\title{
Aggregate Interference Power Characterization for Directional Beamforming Wireless Networks
}

\author{
Ayman T. Abusabah ${ }^{\dagger \ddagger}$ and Rodolfo Oliveira ${ }^{\dagger \ddagger}$ \\ ${ }^{\dagger}$ Departamento de Engenharia Electrotécnica, Faculdade de Ciências e Tecnologia, FCT, \\ Universidade Nova de Lisboa, 2829-516 Caparica, Portugal \\ ${ }_{\text {IT, Instituto de Telecomunicações, Portugal }}$ \\ emails: a.sabah@campus.fct.unl.pt, rado@ fct.unl.pt
}

\begin{abstract}
In this paper, we characterize the aggregate interference power in directional millimeter-wave communications, capturing the effect of beamforming. The analysis considers a general distance-based path loss with Rayleigh and Rician fading channels and a sectored antenna model. Moreover, the nodes are uniformly distributed over a circular or annular area centered at the receiver. The main contribution of the paper is the derivation of the moment generating function (MGF) of the aggregate interference power. The MGF is adopted in a moment-based approximation to a Gamma distribution and used as a model of the aggregate interference power. Several simulations confirm the effectiveness of the proposed approximation for different scenarios, highlighting the effect of directional communications on the aggregate interference power.

Keywords-Directional Beamforming, Millimeter-Wave Communications, Aggregate Interference, Stochastic Geometry Modeling, Performance Analysis.
\end{abstract}

\section{INTRODUCTION}

Besides the greater spectrum availability that millimeterwave (mmWave) and terahertz (THz) communications offer, directional communication has an advantage in overcoming the consequent high isotropic path loss while substantially reducing the interference footprint [1], thus improving spatial reuse. Rigorously speaking, directional communication networks will change the conventional concepts about the interference, i.e., as the nodes only focus their beams toward a specific directed spatial channel. With recent advanced signal processing techniques, such as Multi-User Multiple Input Multiple Output (MU-MIMO), the receivers (RXs) are capable of decoding multiple simultaneously transmitted packets in the wireless channel. In these systems, multiple nodes may act as interferers to each other when a packet transmitted by a specific node is decoded at the RX.

The mmWave communications are an effective solution adopted in 5G systems [2] and Wi-Fi [3]. The IEEE 802.11ay standard enables $100 \mathrm{~Gb} / \mathrm{s}$ using MU-MIMO beamforming in unlicensed millimeter-wave bands [3]. IEEE 802.11ay is also being considered for $5 \mathrm{G}$ fixed wireless access [4]. Given the rising importance of mmWave communications, it is important to know the stochastic nature of the interference considering fading effects and beamforming parameters to determine appropriate antenna configurations and avoid performance degradation.
This paper presents a simple model for the interference caused by multiple transmitters (TXs) to a single RX, considering the spatial density of the nodes, the radio propagation conditions, and directional beamforming. In Section II we present the system model and main assumptions. Section III characterizes the aggregate interference power. Numerical and simulated results are used to validate the model and presented in Section IV. Finally, Section V concludes the paper.

\section{A. Related Work}

The characterization of the interference in wireless networks has been a topic of extensive research in the last years [5][8]. The aggregate interference is usually modeled through advanced stochastic geometry techniques [9] that have into account the spatial position of each interferer and its individual radio channel to determine the amount of interference caused to a specific node [5], [6], [10].

In [11] the authors have adopted the concepts of aligned gain and misaligned gain to derive a coverage model for the Signal-to-interference-plus-noise ratio (SINR). The spatial interference caused by multiple simultaneous and uncoordinated transmissions occurring in the mmWave band was studied in [12] to characterize the collision probability as a function of the antenna patterns and the density of the transmitting nodes. [12] presents interesting results to guide upper layers' design, showing that highly directional links lead to a lower collision probability even for high density of TXs. In mmWave communications, the channels are mainly dominated by lineof-sight (LoS) propagation. This has inspired the work in [10] to study the aggregate interference of mmWave communications for $\operatorname{LoS}$ conditions and first-order reflection paths. The authors have considered blockage objects randomly placed in the propagation scenario and the cumulative distribution function $(\mathrm{CDF})$ of the aggregate interference was derived through the Laplace transform. The authors in [13] have also considered the effect of non-binary object blockage in the aggregate interference, by assuming that a single obstacle can cause a partial blockage. The partial blockage is studied in detail through the derivation of the moment generating function (MGF) of the aggregate interference. Apart from the directivity of the directional antennas and the blockage effect, the work in [14] has also considered molecular absorption in the interference model. The modeling of interference in 
cellular MIMO beamforming mmWave communications was also tackled in [15], by considering two models (inverse Gaussian and the inverse Weibull) and a mixture of them. Using stochastic geometry tools and practical data, the work in [16] studied analytical models for interference in mmWave communications of vehicles moving on side lanes of the highway and urban scenarios, concluding that 2D models can be effectively adopted to model the aggregate interference.

\section{B. Contributions}

This work characterizes the aggregate interference power caused by multiple interferers in directional beamforming networks. The proposed system model considers path loss and two types of small-scale fading effects (Rayleigh and Rician fading). The network model considers that the nodes are uniformly distributed over a circular or annular region centered at the RX. Thereafter, we derive the MGF of the aggregate interference power. The method of moments is adopted to approximate the aggregate interference power as a Gamma distribution. The novelty of our work is the approach adopted in the characterization of the aggregate interference power, the assumption of beamforming and two types of small-scale fading channels, and its validation through simulation results. When compared to related works, the benefits of the proposed work are the simplicity of the approach, which provides a closed-form for the two moments required to approximate the aggregate interference power, apart from the high accuracy and low computational complexity of the model.

\section{SYSTEM MODEL AND ASSUMPTIONS}

\section{A. Network Model}

We consider a scenario where nodes are distributed according to a homogeneous Poisson Point Process (PPP) $\Pi$ with density $\lambda$ over an annular region with an inner radius, $R_{\mathcal{I}}$, and an outer radius, $R_{\mathcal{O}}$, i.e., $R_{\mathcal{I}} \leq r \leq R_{\mathcal{O}}$. For the particular case of $R_{\mathcal{I}}=0$ a circular region is considered. Without loss of generality, we condition on a reference RX at the origin, which according to Slivnyak's theory in stochastic geometry [9], yields to a homogeneous PPP with the corresponding density $\lambda$. At a given time slot, we assume that one or more TXs cause interference to the RX. We consider a single-hop transmission only, with the same transmission power for all nodes. Thus, the total interference seen at the reference RX is the summation of the signals from all transmitting nodes.

\section{B. Gain Pattern and Beamforming Modeling}

All nodes are assumed to be equipped with antenna arrays for performing directional beamforming. We adopt a sectorized antenna model as depicted in Fig. 1, to represent the gain pattern $G^{T}$ and $G^{R}$ at the TX and RX, respectively, as follows

$$
G_{T, R}(\theta)=\left\{\begin{array}{ll}
G_{T, R}^{\max }, & \text { if }|\theta| \leq \omega / 2 \\
G_{T, R}^{\min }, & \text { if }|\theta| \geq \omega / 2
\end{array},\right.
$$

where $G^{\max }$ and $G^{\text {min }}$ are the gains of main and side lobes, respectively, defined by beamwidth $\omega \in(0,2 \pi)$ and the boresight angle direction $\theta \in(-\pi, \pi)$. Without loss of generality, we assume that all nodes are on the same horizontal plane, i.e., no variation in beam pattern over the elevation angle, and we work with the normalized 2-D pattern. While the antenna modeling might look quite simplified, it was adopted in [12] for establishing innovative MAC policies and has been adopted in other works [7], [8].

The RX at the origin and its corresponding TX are assumed to steer their gain patterns toward each other. Assuming perfect estimation of angles of arrival and no alignment errors, the directivity gain of the intended link is $G_{\mathcal{I}}=G_{T}^{\max } G_{R}^{\max }$. The beam orientation of interferers is assumed to be uniformly distributed in $[-\pi, \pi]$. Since the output of (1) is $G^{\max }$ or $G^{\text {min }}$ over $[-\pi, \pi]$, the resulting gain distribution of an $i$-th interfering node, $G_{i, \mathcal{I}}(\theta)$, observed at the RX is discrete and its probability density function (PDF) is given by

$$
f_{G_{i, \mathcal{I}}}(g)=p \delta\left(g-G^{\max }\right)+\bar{p} \delta\left(g-G^{\min }\right),
$$

where $p=\omega / 2 \pi$ is the main lobe hit rate and $\bar{p}=1-p$ is the main lobe miss rate between interferers and the RX.

\section{Communication Model}

We consider a large-scale, distance-based path loss and small-scale fading for modeling the wireless channel. Therefore, the received power caused by an $i$-th node at the RX is given by

$$
P_{i}=P_{t} H_{i} G_{i, \mathcal{I}} G_{\mathcal{I}, i}\left(r_{i}+1\right)^{-\alpha},
$$

where $P_{t}$ is the same transmitted power across all nodes. We consider both Rayleigh and Rician small-scale fading channels in which $H_{i}$ is drawn from a Gamma distribution as will be shown later. Moreover $G_{i, \mathcal{I}}$ and $G_{\mathcal{I}, i}$ represent the gain of an $i$-th TX and the gain of the RX, respectively. $\alpha>2$ is the large-scale path loss coefficient, and $r_{i}$ is the distance between an $i$-th TX and the RX. We highlight that $\left(r_{i}+1\right)$ is assumed in (3) to eliminate the singularity at $r_{i}=0$ and consider that the received power is at most equal to the received power at distance 0 [17].

The aggregate interference power caused to the $\mathrm{RX}$ is formulated as

$$
I_{o}=\sum_{i=1}^{N} P_{i}, i \in \Pi,
$$

where $N$ is a $\mathrm{RV}$ characterized by a homogeneous PPP that represents the number of active TXs over the region represented by the area $A=\pi\left(R_{\mathcal{O}}^{2}-R_{\mathcal{I}}^{2}\right)$. In the next section, we describe the required steps for characterizing the distribution of the aggregate interference power.

\section{CHARACTERIZATION OF AGGREGATE INTERFERENCE POWER}

Since the aggregate interference power, denoted by $I_{o}$, represents the summation of the power generated by each node, we first start our analysis by characterizing the interference power caused by an individual node. According to (3), $P_{i}$ can be seen as a product of the RVs $H_{i}, G_{i}$, and $r_{i}^{-\alpha}$, where $G_{i}=G_{i, \mathcal{I}} G_{\mathcal{I}, i}$ is the directivity gain of an $i$-th interfering 


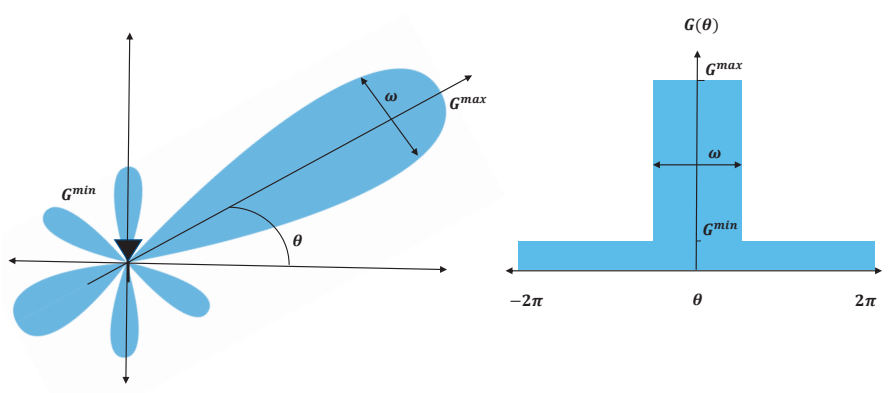

Fig. 1. Beamforming sectored antenna model.

link. The following steps are performed for approximating $P_{i}$ and consequently $I_{o}$ :

1) We show that $H_{i}$ can be represented as a Gamma distribution for both Rayleigh and Rician fading channels. Then, we characterize the distribution of $S_{i}$ as a ratio between RVs $H_{i}$ and $\left(r_{i}+1\right)^{\alpha}$.

2) We characterize the distribution of $G_{i}$, then, we find the distribution of $P_{i}$ as a product between $S_{i}$ and $G_{i}$.

3) Finally, we derive the MGF of $I_{o}$ and we use moment matching to approximate $I_{O}$ as a Gamma distribution.

\section{A. Characterization of Random Variable $S_{i}$}

The Rayleigh channel can describe the stochastic fading when there is no LoS signal. When the channel is a Rayleigh distributed, $H_{i}$ can be drawn from an exponential distribution with mean $\frac{1}{\mu}$, therefore, it can be expressed by a Gamma distribution with the shape parameter, $k=1$, and the scale parameter, $\theta=\frac{1}{\mu}$, as follows

$$
H_{i} \sim \operatorname{Exp}(\mu) \sim \operatorname{Gamma}(k, \theta) .
$$

On the other hand, the Rician fading channel is parameterized through $K$ and $\Omega$ where $K$ represents the quotient between the power in the LoS component and the power in the other nonLoS components, and $\Omega$ represents the total power from both components. Then, the received signal amplitude is Rician distributed with parameters $\nu^{2}=\frac{K \Omega}{1+K}$ and $\sigma^{2}=\frac{\Omega}{2(1+K)}$. $K_{d B}=10 \log _{10}(K)$ is the decibels representation of $K$. By definition, if $X \sim \operatorname{Rice}(\nu, \sigma)$, then $\left(\frac{X}{\sigma}\right)^{2}$ follows a non-central Chi-squared distribution with 2 degrees of freedom and noncentrality parameter $\left(\frac{\nu}{\sigma}\right)^{2}$. Consequently, moment matching can then be used to obtain a simplified Gamma approximation for $H_{i}$ as follows

$$
H_{i} \sim \operatorname{Gamma}(k, \theta),
$$

where $k$ and $\theta$ are the shape and scale parameters, respectively, given by [18]

$$
k=\frac{\left(\nu^{2}+2 \sigma^{2}\right)^{2}}{4 \sigma^{2}\left(\nu^{2}+\sigma^{2}\right)}, \theta=\frac{4 \sigma^{2}\left(\nu^{2}+\sigma^{2}\right)}{\left(\nu^{2}+2 \sigma^{2}\right)^{2}} .
$$

Lets assume that $L_{i}=\left(r_{i}+1\right)^{\alpha}$, so the normalized channel gain, $S_{i}$, can be written as

$$
S_{i}=\frac{H_{i}}{L_{i}} .
$$

Since the nodes are uniformly distributed over $A=\pi\left(R_{\mathcal{O}}^{2}-\right.$ $R_{\mathcal{I}}^{2}$ ), the probability that an interfering node is positioned at distance $r$ from the RX is given by the $\mathrm{CDF} F_{R}(r)=$ $\frac{\left(r^{2}-R_{I}^{2}\right)}{\left(R_{\mathcal{O}}^{2}-R_{\mathcal{I}}^{2}\right)}$, which yields to the PDF $f_{R}(r)=\frac{2 r}{\left(R_{\mathcal{O}}^{2}-R_{\mathcal{I}}^{2}\right)}$. Consequently, the CDF and PDF of $L_{i}$ are respectively stated as follows

$$
\begin{gathered}
F_{L_{i}}(l)=\frac{\left(l^{\frac{1}{\alpha}}-1\right)^{2}-R_{\mathcal{I}}^{2}}{\left(R_{\mathcal{O}}^{2}-R_{\mathcal{I}}^{2}\right)}, \quad \text { for } R_{\mathcal{I}}^{\alpha} \leq l \leq R_{\mathcal{O}}^{\alpha}, \\
f_{L_{i}}(l)=\frac{2\left(l^{\frac{1}{\alpha}}-1\right) l^{\frac{1}{\alpha}-1}}{\alpha\left(R_{\mathcal{O}}^{2}-R_{\mathcal{I}}^{2}\right)}, \quad \text { for } R_{\mathcal{I}}^{\alpha} \leq l \leq R_{\mathcal{O}}^{\alpha} .
\end{gathered}
$$

Assuming that the RV $H_{i}$ is independent of the $\mathrm{RV} L_{i}$, then, the PDF of $S_{i}$ is simply given by the ratio distribution as follows

$$
f_{S_{i}}(s)=\int_{-\infty}^{\infty}|l| f_{H_{i}}(l s) f_{L_{i}}(l) d l .
$$

The integral in (10) can be solved by replacing $f_{L_{i}}(l)$ by (9b) and $f_{H_{i}}(l s)$ by (5) or (6), yields to

$$
f_{S_{i}}(s)=\int_{R_{\mathcal{I}}^{\alpha}}^{R_{\mathcal{O}}^{\alpha}}|l| \frac{(l s)^{k-1} e^{\frac{-l s}{\theta}}}{\Gamma(k) \theta^{k}} \frac{2\left(l^{\frac{1}{\alpha}}-1\right) l^{\frac{1}{\alpha}-1}}{\alpha\left(R_{\mathcal{O}}^{2}-R_{\mathcal{I}}^{2}\right)} d l,
$$

where $k$ and $\theta$ are defined as in (5) for the Rayleigh channel and as in (6) for the Rician channel. After solving the integral in (11), the PDF of $S_{i}$ is written as

$$
\begin{aligned}
& f_{S_{i}}(s)=\left(s^{\frac{1}{\alpha}}\left(\Gamma\left[k+\frac{1}{\alpha}, \frac{R_{\mathcal{I}}^{\alpha} s}{\theta}\right]-\Gamma\left[k+\frac{1}{\alpha}, \frac{R_{\mathcal{O}}^{\alpha} s}{\theta}\right]\right)+\theta^{\frac{1}{\alpha}}\right. \\
& \left.\times\left(\Gamma\left[k+\frac{2}{\alpha}, \frac{R_{\mathcal{O}}^{\alpha} s}{\theta}\right]-\Gamma\left[k+\frac{2}{\alpha}, \frac{R_{\mathcal{I}}^{\alpha} s}{\theta}\right]\right)\right) \frac{2 s^{-\frac{2+\alpha}{\alpha}} \theta^{\frac{1}{\alpha}}}{\alpha\left(R_{\mathcal{I}}^{2}-R_{\mathcal{O}}^{2}\right) \Gamma(k)},
\end{aligned}
$$

where $\Gamma[a, z]$ is the Incomplete Gamma function defined as $\int_{z}^{\infty} t^{a-1} e^{-t} d t$.

\section{B. Characterization of Random Variable $P_{i}$}

The RV $P_{i}$ represents a product between the RVs $S_{i}$ and $G_{i}$. Actually, $G_{i}$ can be seen as a product of two random gains, $G_{i, \mathcal{I}}$ and $G_{\mathcal{I}, i}$, where each follows the PDF in (2). Intuitively, the possible set of outputs for $G_{i}$ are $\left\{G^{\max ^{2}}, G^{\text {min }^{2}}\right.$, $\left.G_{\max } G_{\min }\right\}$ with probabilities $\left\{p^{2}, \bar{p}^{2}, 2 p \bar{p}\right\}$, respectively. Therefore, the PDF of $G_{i}$ can be expressed as follows

$$
\begin{aligned}
f_{G_{i}}(g)= & p^{2} \delta\left(g-G^{\text {max }^{2}}\right)+\bar{p}^{2} \delta\left(g-G^{\text {min }^{2}}\right) \\
& +2 p \bar{p} \delta\left(g-G^{\text {max }} G^{\text {min }}\right),
\end{aligned}
$$

where $p^{2}$ represents the probability that the TX main lobe and RX main lobe hit each other, $\bar{p}^{2}$ represents the probability that the TX main lobe and RX main lobe miss each other, and $2 p \bar{p}$ represents the probability that one hits and the other misses.

The PDF of $P_{i}$ can then be found by the classical product distribution as follows

$$
f_{P_{i}}(x)=\int_{-\infty}^{\infty} \frac{1}{|g|} f_{G_{i}}(g) f_{S_{i}}(x / g) d g .
$$


This integral can be solved by replacing $f_{G_{i}}(g)$ and $f_{S_{i}}(x / g)$ by (13) and (12), respectively, obtaining

$$
\begin{aligned}
& f_{P_{i}}(x)=\frac{p^{2}}{G^{\max ^{2}}} f_{S_{i}}\left(\frac{x}{G^{\text {max }}}\right)+\frac{\bar{p}^{2}}{G^{\min ^{2}}} f_{S_{i}}\left(\frac{x}{G^{\min ^{2}}}\right) \\
& +\frac{2 p \bar{p}}{G^{\min } G^{\max }} f_{S_{i}}\left(\frac{x}{G^{\min } G^{\max }}\right) .
\end{aligned}
$$

Consequently, the MGF of $P_{i}$, defined as $\Phi_{P_{i}}(s)=$ $E\left[e^{s P_{i}}\right]$, where $E[\cdot]$ denotes the expectation, is represented in (16) where $\varrho_{1}(x)={ }_{2} \mathrm{~F}_{1}\left(k, \frac{-1}{\alpha}, \frac{-1+\alpha}{\alpha}, x\right), \varrho_{2}(x)=$ ${ }_{2} \mathrm{~F}_{1}\left(k, \frac{-2}{\alpha}, \frac{-2+\alpha}{\alpha}, x\right)$ and ${ }_{2} \mathrm{~F}_{1}(a, b, c, z)$ is the Gauss Hypergeometric function [19].

\section{Characterization of Random Variable $I_{o}$}

According to (4), the RV $I_{o}$ represents the summation of $N$ independent $\operatorname{RVs}\left(I_{o}=P_{1}+P_{2}+\ldots+P_{N}\right)$. As mentioned before, $N$ follows a homogeneous PPP distribution where

$$
P[N=n]=\frac{(\lambda|A|)^{n}}{n !} e^{-\lambda|A|}
$$

Since $P_{i}$ are independent, then for a specific number of active interferers $n$, we can write

$$
\Phi_{I_{o} \mid n}(s)=\Phi_{P_{1}}(s) \times \Phi_{P_{2}}(s) \cdots \times \Phi_{P_{n}}(s)=\left(\Phi_{P_{i}}(s)\right)^{n} .
$$

Using the law of total probability, the PDF of the aggregate interference $I_{o}$ can be expressed as

$$
f_{I_{o}}(j)=\sum_{n=0}^{\infty} f_{I_{o}}(j \mid N=n) P[N=n] .
$$

Thus, the MFG of the aggregate interference, $\Phi_{I_{o}}(s)$, can be written as

$$
\begin{aligned}
\Phi_{I_{o}}(s) & =E\left[e^{s I_{o}}\right]=\sum_{n=0}^{\infty} P[N=n] \int_{-\infty}^{\infty} e^{s j} f_{I_{o}}(j \mid N=n) \\
& =\sum_{n=0}^{\infty} P[N=n] \Phi_{I_{o} \mid n}(s)=\sum_{n=0}^{\infty} \frac{\left(\lambda|A| \Phi_{P_{i}}(s)\right)^{n}}{n !} e^{-\lambda|A|} .
\end{aligned}
$$

As $e^{x}=\sum_{n=0}^{\infty}\left(x^{n}\right) / n$ !, (20) can be expressed as follows

$$
\Phi_{I_{o}}(s)=e^{\lambda|A|\left(\Phi_{P_{i}}(s)-1\right)} .
$$

We can obtain $k$-th moment of $I_{o}$ from (21), i.e., $\mathrm{E}\left[I_{o}^{k}\right]=$ $\left.\frac{d^{k}}{d s^{k}} \Phi_{I_{o}}(s)\right|_{s=0}$. For the approximation of $I_{o}$, the moments can then be matched with the respective moments of a given distribution. Empirical data was used in a maximum loglikelihood estimation process to determine the parameters of the known distributions, where the Gamma distribution achieved the highest accuracy in representing the aggregate interference. This observation is confirmed by [5], where it is shown that aggregate interference due to large-scale and smallscale fading can be approximated by a Gamma distribution, although considering omnidirectional antennas.

Therefore, the PDF of $I_{o}$ can be written as follows

$$
f_{I_{o}}(z)=\frac{z^{k_{o}-1} e^{\frac{-z}{\theta_{o}}}}{\theta_{o}^{k_{o}} \Gamma\left(k_{o}\right)}, \quad \text { for } z>0,
$$

where the shape and scale parameters, $k_{o}$ and $\theta_{o}$, respectively, are given by

$$
k_{o}=\frac{\mathrm{E}\left[I_{o}\right]^{2}}{\mathrm{E}\left[I_{o}^{2}\right]-\mathrm{E}\left[I_{o}\right]^{2}}, \quad \theta_{o}=\frac{\mathrm{E}\left[I_{o}^{2}\right]-\mathrm{E}\left[I_{o}\right]^{2}}{\mathrm{E}\left[I_{o}\right]},
$$

with $\mathrm{E}\left[I_{o}\right]$ and $\mathrm{E}\left[I_{o}^{2}\right]$ given in (24a) and (24b), respectively.

\section{PERFORMANCE ANALYSIS}

This section presents numerical results based on our previous analysis and provides some interpretations. The numerical results obtained with the model are compared with the results obtained through Monte Carlo simulation results $\left(10^{6}\right.$ realizations of the stochastic process were run) to evaluate the accuracy of modeling the aggregate interference power in directional communication. The network and channel parameters are listed in Table I, unless otherwise specified.

TABLE I

NETWORK AND CHANNEL PARAMETERS

\begin{tabular}{|c|c||c|c|}
\hline Symbol & Value & Symbol & Value \\
\hline$P_{t}$ & $1 \mathrm{~mW}$ & $R_{\mathcal{I}}, R_{\mathcal{O}}$ & $0 \mathrm{~m}, 5 \mathrm{~m}$ \\
\hline$\mu$ & 2 & $\lambda$ & 50 nodes $/ \mathrm{m}^{2}$ \\
\hline$K_{d B}$ & 10 & $G^{\text {max }}$ & 1 \\
\hline$\Omega$ & $1 \mathrm{~mW}$ & $G^{\text {min }}$ & $\{0.2,0.3\}$ \\
\hline$\alpha$ & 4 & $\omega$ & $\left\{45^{\circ}, 60^{\circ}\right\}$ \\
\hline
\end{tabular}

The CDFs of the aggregate interference power are illustrated in Fig. 2 considering a Rayleigh fading channel with different gain levels of the beam side lobes and different beamwidths. The "simulation" curves were obtained through Monte Carlo simulation while "theoretical" curves were obtained with the computation of the CDF associated with the PDF in (22). The similarity comparison between the simulated data and the theoretical derivations indicates the high accuracy of the proposed approximation. Besides, the results reflect the incremental effect on the interference power due to the increase

$$
\begin{aligned}
\Phi_{P_{i}}(s) & =\frac{1}{\left(R_{\mathcal{I}}^{2}-R_{\mathcal{O}}^{2}\right)}\left(p^{2} R_{\mathcal{I}}^{2} \varrho_{2}\left(G^{\text {max } 2} R_{\mathcal{I}}^{-\alpha} s \theta\right)+\bar{p}^{2} R_{\mathcal{I}}^{2} \varrho_{2}\left(G^{\text {min } 2} R_{\mathcal{I}}^{-\alpha} s \theta\right)+2 p \bar{p} R_{\mathcal{I}}^{2} \varrho_{2}\left(G^{\text {min }} G^{\text {max }} R_{\mathcal{I}}^{-\alpha} s \theta\right)-p^{2} R_{\mathcal{O}}^{2} \varrho_{2}\left(G^{\text {max } 2} R_{\mathcal{O}}^{-\alpha} s \theta\right)\right. \\
& -\bar{p}^{2} R_{\mathcal{O}}^{2} \varrho_{2}\left(G^{\text {min } 2} R_{\mathcal{O}}^{-\alpha} s \theta\right)-2 p \bar{p} R_{\mathcal{O}}^{2} \varrho_{2}\left(G^{\text {max }} G^{\text {min }} R_{\mathcal{O}}^{-\alpha} s \theta\right)-2 p^{2} R_{\mathcal{I}} \varrho_{1}\left(G^{\text {max } 2} R_{\mathcal{I}}^{-\alpha} s \theta\right)-2 \bar{p}^{2} R_{\mathcal{I}} \varrho_{1}\left(G^{\text {min } 2} R_{\mathcal{I}}^{-\alpha} s \theta\right) \\
& \left.-4 p \bar{p} R_{\mathcal{I} \varrho_{1}}\left(G^{\text {max }} G^{\text {min }} R_{\mathcal{I}}^{-\alpha} s \theta\right)+2 p^{2} R_{\mathcal{O}} \varrho_{1}\left(G^{\text {max } 2} R_{\mathcal{O}}^{-\alpha} s \theta\right)+2 \bar{p}^{2} R_{\mathcal{O}} \varrho_{1}\left(G^{\text {min } 2} R_{\mathcal{O}}^{-\alpha} s \theta\right)+4 p \bar{p} R_{\mathcal{O}} \varrho_{1}\left(G^{\text {max }} G^{\text {min }} R_{\mathcal{O}}^{-\alpha} s \theta\right)\right)
\end{aligned}
$$




$$
\begin{aligned}
\mathrm{E}\left[I_{o}\right]= & \frac{2 A e^{\frac{A\left(-R_{\mathcal{I}}-R_{\mathcal{O}}+(p+\bar{p})^{2}\left(-2+R_{\mathcal{I}}+R_{\mathcal{O}}\right)\right) \lambda}{R_{\mathcal{I}}+R_{\mathcal{O}}}} k\left(R_{\mathcal{I}} R_{\mathcal{O}}\right)^{-\alpha} \theta \lambda\left(p G^{\text {max }}+\bar{p} G^{m i n}\right)^{2}}{\left(R_{\mathcal{I}}^{2}-R_{\mathcal{O}}^{2}\right)(-2+\alpha)(-1+\alpha)}\left(R_{\mathcal{I}}^{2} R_{\mathcal{O}}^{\alpha}+R_{\mathcal{I}}^{1+\alpha} R_{\mathcal{O}}(-2+\alpha)-R_{\mathcal{I}}^{\alpha} R_{\mathcal{O}}^{2}(-1+\alpha)\right) \\
\mathrm{E}\left[I_{o}^{2}\right]= & \frac{A e^{\frac{A\left(-R_{\mathcal{I}}-R_{\mathcal{O}}+(p+\bar{p})^{2}\left(-2+R_{\mathcal{I}}+R_{\mathcal{O}}\right)\right)^{\lambda}}{R_{\mathcal{I}}+R_{\mathcal{O}}}} k\left(R_{\mathcal{I}} R_{\mathcal{O}}\right)^{-2 \alpha} \theta^{2} \lambda}{\left(R_{\mathcal{I}}^{2}-R_{\mathcal{O}}^{2}\right)^{2}(-1+\alpha)^{2}}\left(\frac{4 A k \lambda\left(p G^{m a x}+\bar{p} G^{m i n}\right)^{4}}{(-2+\alpha)^{2}}\left(R_{\mathcal{I}}^{2} R_{\mathcal{O}}^{\alpha}+R_{\mathcal{I}}^{\alpha} R_{\mathcal{O}}\left(R_{\mathcal{O}}+R_{\mathcal{I}}(-2+\alpha)-R_{\mathcal{O}} \alpha\right)\right)^{2}\right. \\
& \left.-\frac{(1+k)(-1+\alpha)\left(R_{\mathcal{I}}^{2}-R_{\mathcal{O}}^{2}\right)\left(G^{\text {max } 2} p+G^{\text {min } 2} \bar{p}\right)^{2}}{-1+2 \alpha}\left(R_{\mathcal{I}}^{2}+R_{\mathcal{O}}^{2 \alpha}+R_{\mathcal{I}}^{2 \alpha} R_{\mathcal{O}}\left(R_{\mathcal{O}}+2 R_{\mathcal{I}}(-1+\alpha)-2 R_{\mathcal{O}} \alpha\right)\right)\right)
\end{aligned}
$$

in the side lobes gain $\left(G^{\text {min }}=\{0.2,0.3\}\right.$ were adopted in the comparison). On the other hand, the results show that the interference power decreases when adopting small $\omega$ values (narrower beams), due to the concentration of higher power in specific and smaller spatial regions.

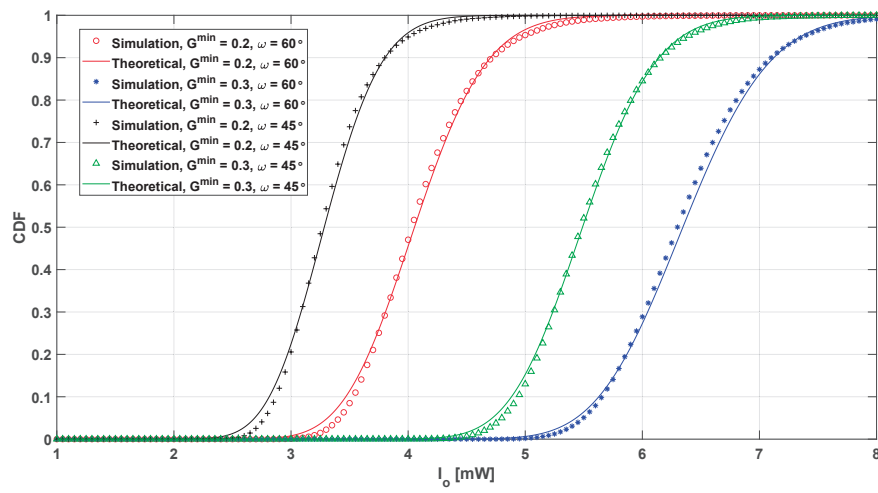

Fig. 2. CDFs of the aggregate interference power $I_{o}$ considering a Rayleigh fading channel for $\mu=2$.

Fig. 3 shows the CDFs of the aggregate interference power when considering a Rician fading channel. Once again the same conclusions can be taken with regards to the incremental effect on the interference power due to the increase in the side lobes gain and the decrease of the power as the beams become narrower. The simulated and theoretically approximated CDFs in Fig. 3 exhibit better matching compared to the CDFs in Fig. 2. From both results in Fig. 2 and Fig. 3 we conclude that although the proposed modeling methodology is quite simple, the approximated CDF is close to the results obtained through simulation and can thus be adopted as an accurate solution to represent the aggregate interference for PPP networks.

\section{CONCLUSiOnS}

This work characterizes the aggregate interference power considering the effect of beamforming when the nodes are distributed according to a homogeneous PPP. In addition to the large-scale path loss, the proposed model considers two types of small-scale fading channels, Rayleigh and Rician. The proposed approach and derivations were validated through simulations, by considering different channel and gain pattern

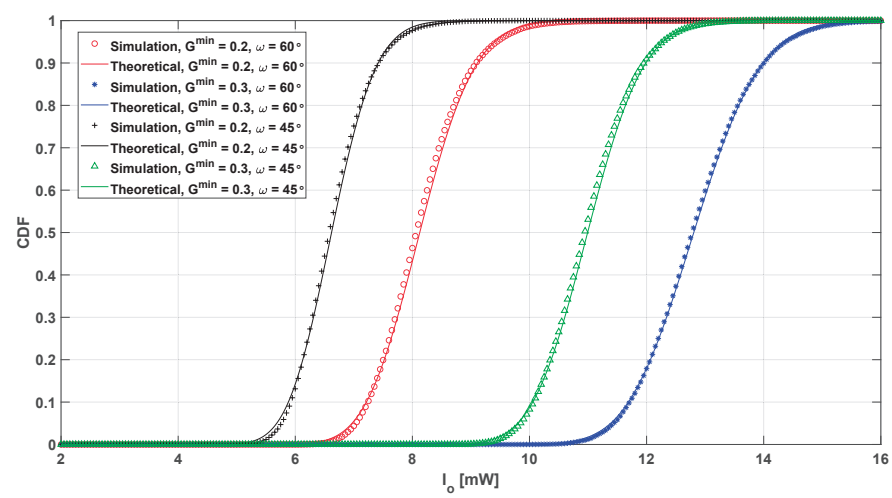

Fig. 3. CDFs of the aggregate interference power $I_{O}$ considering a Rician fading channel: $K_{d B}=10, \nu=0.9535, \sigma=0.2132$.

parameters, confirming the effectiveness and the accuracy of the proposed approximation.

Finally, we highlight that beamforming will play a major role in vehicular communications where the problem becomes far more challenging in dynamic channel conditions. The assumption of dynamic or mobile interferers will be studied in a complementary way to the study we present, being a subject to be addressed in future works.

\section{ACKNOWLEDGEMENTS}

This work has received funding from the European Union's Horizon 2020 research and innovation programme under the Marie Skłodowska-Curie ETN TeamUp5G, grant agreement No. 813391, and partially supported by the projects CoSHARE (LISBOA-01-0145-FEDER0307095 - PTDC/EEITEL/30709/2017), and UIDB/50008/2020.

\section{REFERENCES}

[1] H. Shokri-Ghadikolaei, C. Fischione, G. Fodor, P. Popovski, and M. Zorzi. Millimeter wave cellular networks: A mac layer perspective. IEEE Trans. Commun., 63(10):3437-3458, 2015.

[2] Z. Gao, L. Dai, D. Mi, Z. Wang, M. A. Imran, and M. Z. Shakir. Mmwave massive-mimo-based wireless backhaul for the $5 \mathrm{~g}$ ultra-dense network. IEEE Wirel. Commun, 22(5):13-21, 2015.

[3] Y. Ghasempour, C. R. C. M. da Silva, C. Cordeiro, and E. W. Knightly. Ieee 802.11ay: Next-generation $60 \mathrm{ghz}$ communication for $100 \mathrm{gb} / \mathrm{s} \mathrm{wi-}$ fi. IEEE Commun. Mag., 55(12):186-192, 2017.

[4] K. Aldubaikhy, W. Wu, N. Zhang, N. Cheng, and X. Shen. mmwave ieee 802.11ay for 5g fixed wireless access. IEEE Wirel. Commun, 27(2):8895, 2020. 
[5] Martin Haenggi and Radha Ganti. Interference in large wireless networks. Foundations and Trends in Networking, 3:127-248, 012009.

[6] L. Irio, A. Furtado, R. Oliveira, L. Bernardo, and R. Dinis. Interference characterization in random waypoint mobile networks. IEEE Trans. Wireless Commun., 17(11):7340-7351, 2018.

[7] M. Di Renzo. Stochastic geometry modeling and analysis of multitier millimeter wave cellular networks. IEEE Trans. Wireless Commun. 14(9):5038-5057, 2015.

[8] J. Wildman and P. H. J. Nardelli and M. Latva-aho and S. Weber. On the Joint Impact of Beamwidth and Orientation Error on Throughput in Directional Wireless Poisson Networks. IEEE Trans. Wireless Commun, 13(12):7072-7085, 2014.

[9] F. Baccelli and B. Blaszczyszyn. Stochastic Geometry and Wireless Networks. Delft, The Netherlands: Now Publishers, Sep.2009.

[10] M. Dong and T. Kim. Interference analysis for millimeter-wave networks with geometry-dependent first-order reflections. IEEE Trans. Veh. Technol., 67(12):12404-12409, 2018.

[11] M. Rebato, J. Park, P. Popovski, E. De Carvalho, and M. Zorzi. Stochastic geometric coverage analysis in mmwave cellular networks with realistic channel and antenna radiation models. IEEE Trans. Commun., 67(5):3736-3752, 2019.

[12] S. Singh, R. Mudumbai, and U. Madhow. Interference analysis for highly directional 60-ghz mesh networks: The case for rethinking medium access control. IEEE ACM Trans Netw, 19(5):1513-1527, 2011.

[13] S. Niknam, B. Natarajan, and R. Barazideh. Interference analysis for finite-area $5 \mathrm{~g}$ mmwave networks considering blockage effect. IEEE Access, 6:23470-23479, 2018.

[14] V. Petrov, M. Komarov, D. Moltchanov, J. M. Jornet, and Y. Koucheryavy. Interference and sinr in millimeter wave and terahertz communication systems with blocking and directional antennas. IEEE Trans. Wireless Commun., 16(3):1791-1808, 2017.

[15] H. Elkotby and M. Vu. Interference modeling for cellular networks under beamforming transmission. IEEE Trans. Wireless Commun., 16(8):52015217, 2017.

[16] V. Petrov, J. Kokkoniemi, D. Moltchanov, J. Lehtomaki, M. Juntti, and Y. Koucheryavy. The impact of interference from the side lanes on mmwave/thz band $\mathrm{v} 2 \mathrm{v}$ communication systems with directional antennas. IEEE Trans. Veh. Technol., 67(6):5028-5041, 2018.

[17] J. Venkataraman, M. Haenggi, and O. Collins. Shot noise models for outage and throughput analyses in wireless ad hoc networks. In MILCOM 2006 - 2006 IEEE Military Communications conference, pages 1-7, 2006.

[18] A. T. Abusabah, L. Irio, R. Oliveira, and D. B. da Costa. Approximate distributions of the residual self-interference power in multi-tap fullduplex systems. IEEE Wireless Commun. Lett., pages 1-1, 2020.

[19] M. Abramowitz and I. A. Stegun. Handbook of Mathematical Functions: With Formulas, Graphs, and Mathematical Tables. New York, NY, USA: Dover, 1965. 